\title{
Resuscitative Endovascular Balloon Occlusion of the Aorta in a Trauma Patient with Hypovolemic Shock
}

\author{
Hong Kyung Shin, M.D., Ho-Seong Han, M.D., Ph.D., Taeseung Lee, M.D., Ph.D., Do-Joong Park, M.D., Ph.D., \\ Kyuwhan Jung, M.D., Ph.D., and Kyuseok Kim, M.D., Ph.D.*
}

Department of Surgery; * Department of Emergency Medicine, Seoul National University Bundang Hospital, Seongnam, Korea

\begin{abstract}
Hemorrhagic shock is one of the most common causes of death in patients with multiple trauma and therefore rapid control of bleeding is the main strategy to save these patients. Resuscitative balloon occlusion of the aorta (REBOA) has been applied in several trauma cases and because of the effectiveness of this procedure it has been adopted in the trauma field. Herein, we report the first successful case of REBOA in Korea performed on a 46-year-old man with hemorrhagic shock after a fall from a height of 14-stories. The patient visited our hospital emergency room with hypovolemic shock, we performed Resuscitative Endovascular Balloon Occlusion of the Aorta under bed side blind technique. His vital sign was stabilized after procedure, then we could performed endovascular bleeding control. The patient was discharged on his 33rd in-hospital day without invasive procedure and major scar.
\end{abstract}

Key Words: aortic balloon; hemorrhage; hypovolemic shock; thoracotomy trauma.

Hypovolemic shock with exsanguination is one of the most common causes of death in multiple trauma patients, and is the most common causes of preventable death in severely injured patients.[1] Many reports have suggested that resuscitation without bleeding control makes a good prognosis difficult; rapid surgical treatment or other bleeding control procedures have been emphasized for saving patients experiencing hemorrhagic shock.[2]

Resuscitative balloon occlusion of the aorta (REBOA) has already been described for the treatment of hemorrhagic shock in cases of ruptured abdominal aortic aneurysm, gastrointestinal bleeding, and postpartum hemorrhage.[3] Recently, REBOA has been applied in trauma cases, and because of its effectiveness, it has been adopted in the trauma field.[4] Currently, there is no case of REBOA reported in Korea. Here, we report the first successful emergency room (ER)-based REBOA in a trauma patient experiencing hemorrhagic shock.

\section{Case Report}

A 46-year-old male patient visited the local hospital ER after attempting suicide by jumping from a 14th floor apartment.

Received on December 31, 2014 Revised on March 17, 2015

Accepted on April 3, 2015

Correspondence to: Ho-Seong Han, Department of Surgery, Seoul National University Bundang Hospital, 82 Gumi-ro 173beon-gil, Bundang-gu, Seongnam 463-707, Korea

Tel: +82-31-787-7091, Fax: +82-31-787-4078

E-mail: hanhs@snubh.org

*No potential conflict of interest relevant to this article was reported.
He was transferred to our hospital ER for further treatment. The patient had multiple traumas, including a minimal skull and ulnar fracture, but the most serious injury was a pelvic fracture with internal iliac artery injury. Despite resuscitation with blood transfusion in the previous ER, the patient presented with a drowsy status and an initial blood pressure (BP) of 56/36, necessitating intubation. Extravasation of the 


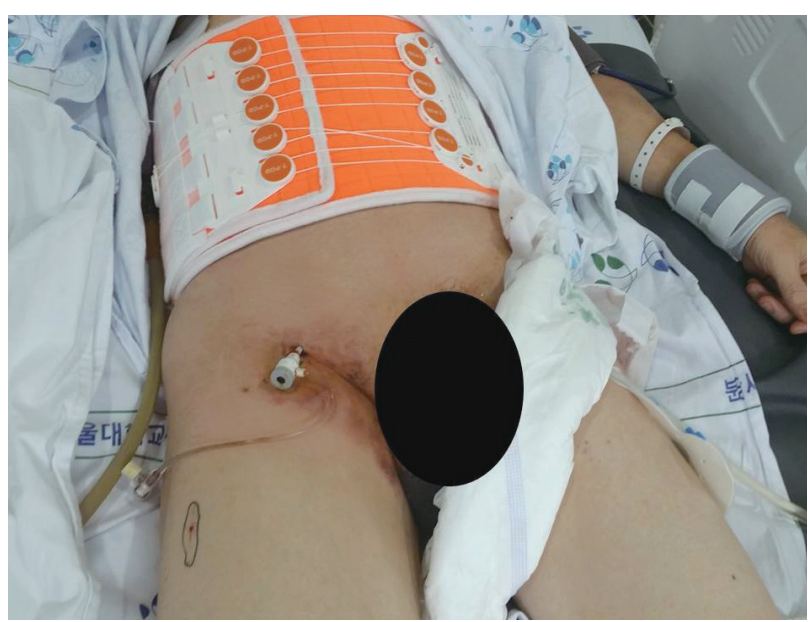

Fig. 1. Inserted $12 \mathrm{Fr}$. sheath for REBOA. REBOA: resuscitative balloon occlusion of the aorta.

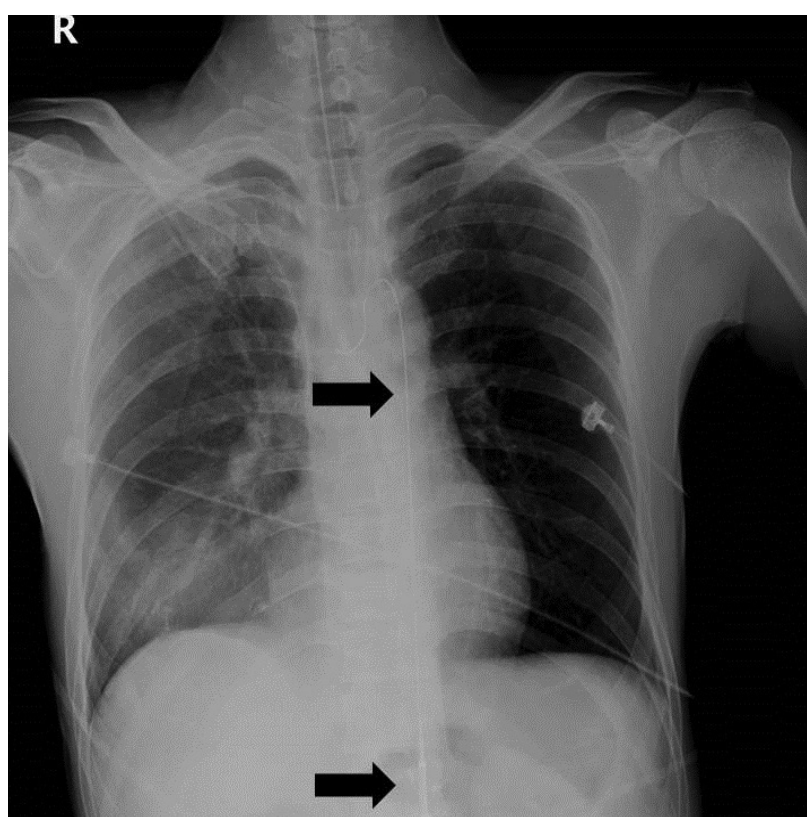

Fig. 2. Correct location of guide wire and balloon in portable $X$ ray film.

internal iliac artery caused by the pelvic bone fracture was detected at the previous hospital with a computed tomography (CT) scan. After identifying the cause of hypovolemic shock-bleeding from the internal iliac artery, we decided to perform REBOA immediately. We had prepared the REBOA kit by the bedside, having received word of the patient's situation from the previous hospital, because the patient was hemodynamically unstable.

The patient's femoral pulse was weak, and after several blind trials, we were able to successfully make a puncture within a few seconds. After inserting the 12 Fr. Sheath (Fig. 1), Lunderquist $^{\circledR}$ aorta guide-wire (Cook Inc. Bloomington, IN, USA) were inserted in the abdominal aorta. After $\operatorname{CODA}^{\circledR}$ aortic balloon (Cook Inc. Bloomington, IN, USA) placement, we verified the location of the guide wire and the balloon with a portable radiography (Fig. 2). The balloon was visualized in the diaphragm-level aorta in the x-ray film, after injection of $200 \mathrm{~cm} 3$ saline. From draping to aortic ballooning, the entire procedure took approximately 15 minutes to complete; a trauma surgeon trained in vascular surgery carried out the procedures. The patient's BP had increased to 90/54 within 7 minutes of ballooning, and a systolic blood pressure (SBP) of above $80 \mathrm{~mm} \mathrm{Hg}$ was maintained.

After ballooning, there was no hypotensive event. We performed intermittent balloon deflation for distal perfusion because the balloon was at the supraceliac level. When deflation affected BP, the balloon was immediately reinflated, allowing BP to gradually increase, and maintain permissive hypotension about $80 \mathrm{~s} \mathrm{mmHg}$ range of SBP.

One hour after ER admission, the patient underwent arterial embolization (AE) of both internal iliac arteries with glue and gelform. After AE, BP was maintained within a normal range, despite balloon removal. No extravasation was noted on a follow-up CT scan, and there was no evidence of ischemia in any internal organs, including the bowel.

On the patient's second day in the hospital, we performed a sheath removal and primary closure of the puncture site of the femoral artery. The patient was transferred into the general ward on his third in-hospital day. On the sixth day, the patient was transferred to the Orthopedic Surgery ward for $\mathrm{OR} / \mathrm{IF}$ (open reduction and internal fixation) of pelvic fracture, and was discharged on his 33rd in-hospital day, after that surgery.

\section{Discussion}

To our knowledge, this is the first successful case report of aortic balloon occlusion for hemorrhage control and resuscitation following trauma in Korea. We have demonstrated that the use of aortic balloon occlusion is feasible, and a promising method to use in hemorrhagic shock to control bleeding, increase vital blood flow, and to improve the chance of survival. 
REBOA for hemorrhagic shock was reported during the Korean War by Hughes.[5] Although not a new technique, REBOA has received a reappraisal because of army surgeons' application of the procedure in managing hemorrhagic shock. The renewed efforts for REBOA stem from the need to develop mechanical or technical adjuncts to improve survival from shock. Previously, efforts in damage control resuscitation have focused on hemostatic dressings, extremity tourniquet devices, and balanced resuscitation using whole blood components and tranexamic acid.[6]

Aortic occlusion in hypovolemic shock can occlude the "unnecessary" portion of the circulation, control bleeding, and divert blood to coronary and cerebral perfusion. Sesma et al[7] and Spence et al.[8] showed that cerebral and myocardial blood flow improved in animal models with constant aortic occlusion during open chest cardiopulmonary resuscitation. Sesma et al showed a $150 \%$ increase in coronary perfusion pressure and $200 \%$ increase in cerebral perfusion pressure. In a similar fashion, Spence et al. showed that although the absolute blood flow reduced by $50 \%$ when aortic balloon occlusion was used compared with that in the control, blood perfusion to the brain and heart during chest compressions improved by $100 \%$.

In recent cases, REBOA was accomplished rapidly and resulted in an average increase in SBP of $55 \mathrm{~mm} \mathrm{Hg}$. The average aortic balloon occlusion time was 18 minutes, which allowed for the performance of hemorrhage control maneuvers.[9] We found that the balloon was easily inserted by the trauma surgeon trained in vascular surgery, offering wide flexibility and control of hemostasis, and thus, facilitating the completion of definitive surgery and allowing successful resuscitation by an emergency physician trained only in endovascular procedures. However, in this case, aortic occlusion time was delayed. We had no choice but to perform on the ER bed without fluoroscopic facility and to wait for portable X-ray and result of that for check the right location of balloon. It needed several minutes.

There have been many reports of compressing the aorta to control bleeding in patients with hemorrhagic shock by externally clamping the aorta.[10] Open aortic crossclamping and REBOA share the same goal, but the latter is less invasive, preventing the potential for additional morbidity in a compromised patient. A recent study has shown that REBOA increased overall survival rates and resulted in fewer ER deaths compared with that to open aortic clamping under resuscitative thoracotomy. Most authors agree that REBOA will replace resuscitative thoracotomy with aortic cross-clamping for proactive and less invasive aortic control in cases of hemorrhagic shock.[11]

We performed blind REBOA in the ER following the patient's ER admission as quickly as possible. The "blind" characteristic of the procedure allows for the performance of this technique in any medical unit, without the need for fluoroscopic guidance. This makes REBOA possible in a secondary hospital lacking an angiographic room as well as in the pre-hospital phase, as in our case. Puncture and catheterization of the femoral artery in a patient with a bulky pelvic hematoma and a low SBP may be difficult, necessitating further systemic training and the development of simplified REBOA kits.[12]

In conclusion, this case is the first record in Korea of a patient who was unresponsive to standard resuscitation maneuvers, and whose bleeding was successfully controlled by REBOA, thus achieving a normal blood pressure, and patient survival to discharge. Patients who develop hemorrhagic shock because of multiple traumas may be successfully resuscitated using intra-aortic balloon occlusion to increase cardio-cerebral perfusion. Further studies are needed to determine the role of constant intra-aortic balloon occlusion during resuscitation in trauma patients.

\section{References}

1) Sauaia A, Moore FA, Moore EE, Moser KS, Brennan R, Read RA, et al: Epidemiology of trauma deaths: a reassessment. J Trauma 1995; 38: 185-93.

2) Willis CD, Cameron PA, Bernard SA, Fitzgerald M: Cardiopulmonary resuscitation after traumatic cardiac arrest is not always futile. Injury 2006; 37: 448-54.

3) Mehta M, Taggert J, Darling RC 3rd, Chang BB, Kreienberg PB, Paty PS, et al: Establishing a protocol for endovascular treatment of ruptured abdominal aortic aneurysms: outcomes of a prospective analysis. J Vasc Surg 2006; 44: 1-8; discussion 8.

4) Stannard A, Eliason JL, Rasmussen TE: Resuscitative endovascular balloon occlusion of the aorta (REBOA) as an adjunct for hemorrhagic shock. J Trauma 2011; 
71: 1869-72.

5) Hughes CW: Use of an intra-aortic balloon catheter tamponade for controlling intra-abdominal hemorrhage in man. Surgery 1954; 36: 65-8.

6) White JM, Cannon JW, Stannard A, Markov NP, Spencer JR, Rasmussen TE: Endovascular balloon occlusion of the aorta is superior to resuscitative thoracotomy with aortic clamping in a porcine model of hemorrhagic shock. Surgery 2011; 150: 400-9.

7) Sesma J, Labandeira J, Sara MJ, Espila JL, Arteche A, Saez MJ: Effect of intra-aortic occlusion balloon in external thoracic compressions during CPR in pigs. Am J Emerg Med 2002; 20:453-62.

8) Spence PA, Lust RM, Chitwood WR Jr, Iida H, Sun YS, Austin EH 3rd: Transfemoral balloon aortic occlusion during open cardiopulmonary resuscitation improves myocardial and cerebral blood flow. J Surg Res 1990; 49:217-21.

9) Brenner ML, Moore LJ, DuBose JJ, Tyson GH, McNutt
MK, Albarado RP, et al: A clinical series of resuscitative endovascular balloon occlusion of the aorta for hemorrhage control and resuscitation. J Trauma Acute Care Surg 2013; 75: 506-11.

10) Ledgerwood AM, Kazmers M, Lucas CE: The role of thoracic aortic occlusion for massive hemoperitoneum. J Trauma 1976; 16: 610-5.

11) Moore LJ: Resuscitative endovascular balloon occlusion of the aorta(REBOA) is a feasible alternative to resuscitative thoracotomy in trauma patients with noncompressible truncal hemorrhage and profound hemorrhagic shock. 73rd Annual Meeting of AAST and Clinical Congress of Acute Care Surgery; 2014 September 10-13; Philadelphia, PA.

12) Martinelli T, Thony F, Declety $P$, Sengel C, Broux C, Tonetti J, et al: Intra-aortic balloon occlusion to salvage patients with life-threatening hemorrhagic shocks from pelvic fractures. J Trauma 2010; 68: 942-8. 